\title{
Supporting Information \\ Machine Learning-Enabled Design and Prediction of Protein Resistance on Self-Assembled Monolayers and Beyond
}

Yonglan Liu ${ }^{1 \zeta}$, Dong Zhang ${ }^{1 \zeta}$, Yijing Tang ${ }^{1}$, Yanxian Zhang ${ }^{1}$, Yung Chang ${ }^{2}$, and Jie Zheng ${ }^{1 *}$

${ }^{1}$ Department of Chemical, Biomolecular, and Corrosion Engineering

The University of Akron, Ohio 44325, USA

${ }^{2}$ Department of Chemical Engineering, R\&D Center for Membrane Technology

Chung Yuan Christian University, Taoyuan, Taiwan

$\zeta$ The authors contribute equally to this work.

*Corresponding Author: zhengj@uakron.edu 
Table S1. Loadings and communalities for the 10 rotated functional groups from factor analysis for the 43 molecular descriptors.

\begin{tabular}{|c|c|c|c|c|c|c|c|c|c|c|c|}
\hline \multirow{2}{*}{$\begin{array}{l}\text { Molecular } \\
\text { Descriptors }\end{array}$} & \multicolumn{10}{|c|}{ Functional Group Number } & \multirow{2}{*}{$\begin{array}{c}\text { Communality } \\
\left(\mathrm{H}^{2}\right)\end{array}$} \\
\hline & 1 & 2 & 3 & 4 & 5 & 6 & 7 & 8 & 9 & 10 & \\
\hline $\mathrm{Me}$ & 0.00 & 0.87 & 0.05 & 0.11 & 0.19 & -0.25 & 0.17 & -0.10 & -0.01 & 0.09 & 0.92 \\
\hline $\mathrm{Mp}$ & 0.06 & -0.05 & 0.73 & -0.05 & 0.66 & 0.04 & 0.03 & 0.00 & -0.04 & 0.03 & 0.98 \\
\hline $\mathrm{Mi}$ & -0.05 & 0.03 & -0.90 & -0.25 & -0.16 & -0.17 & -0.07 & -0.07 & 0.02 & 0.11 & 0.95 \\
\hline GD & -0.21 & -0.30 & -0.38 & -0.70 & -0.10 & -0.17 & -0.03 & -0.08 & -0.12 & -0.13 & 0.84 \\
\hline nTA & 0.72 & 0.48 & 0.01 & 0.06 & 0.05 & 0.08 & -0.04 & 0.05 & 0.41 & 0.16 & 0.96 \\
\hline RBN & 0.22 & 0.04 & -0.17 & 0.75 & -0.02 & 0.21 & 0.32 & 0.21 & 0.22 & -0.04 & 0.88 \\
\hline $\mathrm{RBF}$ & 0.01 & -0.07 & -0.36 & 0.55 & 0.10 & 0.17 & 0.47 & 0.20 & 0.27 & -0.10 & 0.81 \\
\hline $\mathrm{nH}$ & 0.35 & 0.17 & 0.12 & 0.80 & -0.27 & 0.26 & -0.09 & 0.12 & 0.05 & 0.03 & 0.98 \\
\hline $\mathrm{nN}$ & 0.58 & -0.09 & -0.11 & -0.04 & 0.56 & -0.10 & -0.22 & -0.04 & 0.17 & 0.44 & 0.96 \\
\hline N\% & 0.02 & -0.15 & -0.22 & -0.47 & 0.75 & -0.15 & -0.09 & -0.09 & -0.02 & 0.20 & 0.94 \\
\hline $\mathrm{O} \%$ & -0.02 & 0.79 & 0.03 & 0.36 & -0.38 & -0.12 & 0.19 & -0.03 & 0.01 & -0.04 & 0.94 \\
\hline $\mathrm{nCIC}$ & -0.13 & 0.25 & 0.88 & -0.02 & -0.17 & -0.02 & -0.18 & 0.00 & -0.19 & -0.03 & 0.96 \\
\hline Rperim & -0.17 & 0.33 & 0.68 & 0.17 & -0.21 & -0.03 & -0.32 & -0.10 & -0.36 & -0.05 & 0.93 \\
\hline ARR & -0.07 & -0.03 & 0.93 & -0.09 & -0.11 & -0.06 & 0.18 & 0.03 & 0.11 & -0.03 & 0.95 \\
\hline $\mathrm{nCp}$ & 0.34 & 0.27 & 0.00 & 0.06 & -0.28 & 0.19 & 0.59 & 0.38 & -0.34 & 0.03 & 0.93 \\
\hline $\mathrm{nCs}$ & -0.11 & 0.51 & 0.14 & 0.02 & -0.06 & 0.75 & 0.01 & 0.27 & -0.04 & -0.12 & 0.95 \\
\hline $\mathrm{nCt}$ & 0.76 & 0.01 & -0.02 & 0.13 & 0.01 & 0.06 & -0.01 & 0.56 & 0.01 & -0.04 & 0.93 \\
\hline nRCONHR & 0.02 & 0.08 & -0.07 & -0.06 & 0.02 & -0.02 & 0.17 & -0.10 & -0.03 & 0.81 & 0.72 \\
\hline nRCONR2 & 0.85 & -0.02 & -0.02 & 0.11 & 0.06 & -0.06 & -0.18 & -0.04 & 0.13 & 0.23 & 0.85 \\
\hline nRCO & 0.88 & -0.03 & -0.02 & 0.01 & -0.02 & -0.01 & 0.11 & -0.06 & -0.10 & -0.18 & 0.84 \\
\hline nRNH2 & -0.19 & -0.01 & -0.05 & 0.01 & -0.06 & -0.01 & 0.83 & 0.12 & -0.28 & 0.23 & 0.88 \\
\hline nArNH2 & -0.07 & 0.71 & 0.42 & -0.05 & -0.09 & 0.17 & -0.16 & -0.03 & 0.03 & -0.13 & 0.76 \\
\hline nRNHR & 0.19 & -0.36 & -0.19 & 0.02 & 0.08 & -0.09 & -0.73 & -0.10 & 0.27 & -0.16 & 0.86 \\
\hline nRNR2 & -0.12 & -0.25 & -0.02 & -0.01 & -0.13 & -0.09 & -0.50 & 0.20 & -0.17 & 0.13 & 0.44 \\
\hline $\mathrm{nRCN}$ & -0.12 & 0.00 & 0.00 & -0.13 & 0.95 & 0.03 & 0.01 & -0.02 & -0.08 & -0.11 & 0.95 \\
\hline nOHp & -0.11 & 0.56 & 0.06 & 0.05 & -0.16 & 0.08 & 0.04 & 0.73 & -0.12 & -0.13 & 0.94 \\
\hline nROR & -0.28 & 0.15 & -0.02 & 0.83 & -0.16 & -0.09 & 0.05 & -0.18 & -0.19 & -0.17 & 0.92 \\
\hline C-001 & 0.88 & -0.12 & -0.04 & -0.03 & -0.05 & 0.26 & 0.07 & -0.13 & -0.17 & -0.05 & 0.92 \\
\hline C-002 & -0.04 & -0.18 & -0.05 & -0.03 & 0.07 & 0.84 & 0.16 & 0.42 & -0.10 & -0.03 & 0.96 \\
\hline C-003 & 0.92 & 0.00 & 0.01 & 0.16 & 0.02 & 0.00 & -0.06 & 0.03 & 0.03 & 0.00 & 0.88 \\
\hline C-005 & 0.43 & -0.19 & -0.13 & 0.18 & -0.10 & -0.07 & -0.25 & -0.12 & 0.72 & 0.08 & 0.89 \\
\hline C-006 & -0.05 & -0.10 & -0.09 & 0.82 & -0.13 & -0.22 & -0.03 & -0.01 & -0.39 & 0.01 & 0.91 \\
\hline C-041 & -0.07 & -0.07 & -0.04 & -0.03 & 0.00 & -0.04 & -0.10 & -0.03 & 0.56 & -0.02 & 0.34 \\
\hline H-046 & 0.33 & -0.19 & -0.01 & 0.07 & -0.08 & 0.86 & 0.07 & -0.06 & -0.03 & 0.07 & 0.91 \\
\hline H-047 & 0.21 & 0.01 & 0.09 & 0.86 & -0.24 & -0.21 & -0.22 & -0.10 & 0.17 & 0.03 & 0.98 \\
\hline H-051 & 0.33 & -0.11 & -0.07 & -0.29 & 0.37 & 0.03 & 0.19 & -0.23 & -0.33 & -0.26 & 0.61 \\
\hline H-052 & 0.00 & -0.03 & -0.05 & 0.01 & -0.03 & 0.25 & 0.09 & 0.89 & -0.03 & -0.04 & 0.86 \\
\hline O-060 & -0.07 & 0.29 & 0.48 & -0.09 & -0.15 & -0.01 & 0.15 & -0.06 & 0.43 & -0.16 & 0.58 \\
\hline Ui & 0.40 & 0.14 & 0.60 & -0.21 & 0.56 & -0.04 & 0.05 & -0.10 & 0.16 & 0.16 & 0.97 \\
\hline
\end{tabular}




\begin{tabular}{cccccccccccc}
\hline Hy & -0.18 & 0.80 & -0.01 & -0.24 & -0.19 & 0.03 & 0.27 & 0.23 & -0.16 & 0.10 & 0.93 \\
TPSA(NO) & 0.18 & 0.89 & 0.16 & 0.25 & 0.13 & 0.04 & 0.13 & 0.12 & -0.07 & 0.11 & 0.98 \\
ALOGP & -0.10 & -0.68 & 0.42 & -0.15 & 0.01 & 0.49 & 0.02 & 0.04 & 0.14 & -0.17 & 0.97 \\
PDI & 0.10 & -0.63 & 0.67 & 0.08 & 0.08 & -0.03 & -0.04 & -0.11 & -0.07 & 0.22 & 0.94 \\
\hline
\end{tabular}


Table S2. Regression coefficients of molecular descriptors with absolute coefficient greater than 0.3 for the 10 functional group.

\begin{tabular}{|c|c|c|c|c|c|c|c|c|c|}
\hline \multicolumn{2}{|c|}{ Functional Group 1} & \multicolumn{2}{|c|}{ Functional Group 2} & \multicolumn{2}{|c|}{ Functional Group 3} & \multicolumn{2}{|c|}{$\begin{array}{l}\text { Functional } \\
\text { Group } 4\end{array}$} & \multicolumn{2}{|c|}{ Functional Group 5} \\
\hline C-003 & 0.92 & nTA & 0.48 & nArNH2 & 0.42 & $\mathrm{O} \%$ & 0.36 & $\mathrm{nN}$ & 0.56 \\
\hline $\mathrm{nRCO}$ & 0.88 & TPSA(NO) & 0.89 & ALOGP & 0.42 & H-047 & 0.86 & $\mathrm{O} \%$ & -0.38 \\
\hline C-001 & 0.88 & $\mathrm{Me}$ & 0.87 & ARR & 0.93 & nROR & 0.83 & $\mathrm{Mp}$ & 0.66 \\
\hline nRCONR2 & 0.85 & Hy & 0.80 & Mi & -0.90 & C-006 & 0.82 & $\mathrm{Ui}$ & 0.56 \\
\hline $\mathrm{nCt}$ & 0.76 & $\mathrm{O} \%$ & 0.79 & nCIC & 0.88 & $\mathrm{nH}$ & 0.80 & $\mathrm{nRCN}$ & 0.95 \\
\hline nTA & 0.72 & nArNH2 & 0.71 & $\mathrm{Mp}$ & 0.73 & RBN & 0.75 & N\% & 0.75 \\
\hline $\mathrm{nN}$ & 0.58 & ALOGP & -0.68 & Rperim & 0.68 & GD & -0.70 & $\mathrm{H}-051$ & 0.37 \\
\hline Ui & 0.40 & Rperim & 0.33 & PDI & 0.67 & RBF & 0.55 & & \\
\hline $\mathrm{nH}$ & 0.35 & PDI & -0.63 & Ui & 0.60 & N\% & -0.47 & & \\
\hline H- 051 & 0.33 & $\mathrm{nCs}$ & 0.51 & O-060 & 0.48 & & & & \\
\hline H-046 & 0.33 & nRNHR & -0.36 & GD & -0.38 & & & & \\
\hline $\mathrm{nCp}$ & 0.34 & nOHp & 0.56 & RBF & -0.36 & & & & \\
\hline C-005 & 0.43 & & & & & & & & \\
\hline \multicolumn{2}{|c|}{ Functional Group 6} & \multicolumn{2}{|c|}{ Functional Group 7} & \multicolumn{2}{|c|}{ Functional Group 8} & \multicolumn{2}{|c|}{$\begin{array}{c}\text { Functional } \\
\text { Group } 9\end{array}$} & \multicolumn{2}{|c|}{ Functional Group 10} \\
\hline ALOGP & 0.49 & Rperim & -0.32 & $\mathrm{nCt}$ & 0.56 & nTA & 0.41 & $\mathrm{nN}$ & 0.44 \\
\hline H-046 & 0.86 & RBN & 0.32 & C-002 & 0.42 & Rperim & -0.36 & nRCONHR & 0.81 \\
\hline C-002 & 0.84 & RBF & 0.47 & $\mathrm{nCp}$ & 0.38 & O-060 & 0.43 & & \\
\hline $\mathrm{nCs}$ & 0.75 & nRNH2 & 0.83 & H-052 & 0.89 & C-006 & -0.39 & & \\
\hline & & nRNHR & -0.73 & nOHp & 0.73 & H-051 & -0.33 & & \\
\hline & & $\mathrm{nCp}$ & 0.59 & & & $\mathrm{nCp}$ & -0.34 & & \\
\hline & & nRNR2 & -0.50 & & & $\mathrm{C}-005$ & 0.72 & & \\
\hline & & & & & & C-041 & 0.56 & & \\
\hline
\end{tabular}


Table S3. Summary of percentage of variance of 43 molecular descriptors included in the top 10 functional groups.

\begin{tabular}{ccc}
\hline Functional Group Number & Percentage of Variance (\%) & Cumulative Percentage of Variance (\%) \\
\hline 1 & 13.67 & 13.67 \\
2 & 13.58 & 27.25 \\
3 & 12.49 & 39.74 \\
4 & 11.53 & 51.27 \\
5 & 7.95 & 59.22 \\
6 & 6.77 & 65.99 \\
7 & 6.68 & 72.67 \\
8 & 5.77 & 78.44 \\
9 & 5.44 & 83.88 \\
10 & 3.46 & 87.34 \\
\hline
\end{tabular}




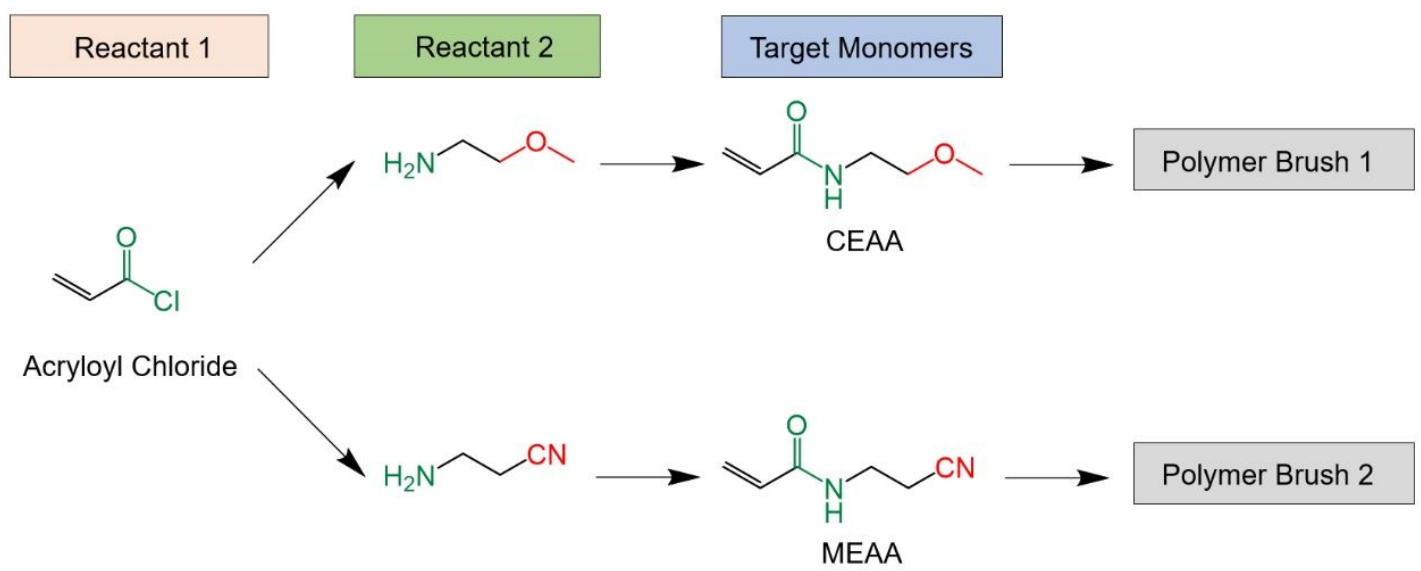

Figure S1. Synthesis procedure of two monomers (CEAA, and MEAA) of designed antifouling materials, which are further used to synthesize polymer brushes. 